\title{
Métodos de pesquisa com usuários: a abordagem etnográfica aplicada ao design
}

\section{User research methods: the ethnographic approach applied to design}

\author{
Uda Flavia Souza Fialho \\ Doutoranda em Design pela Universidade Federal do Rio Grande do Sul (UFRGS) - udaflavia@gmail.com \\ Júlio Carlos de Souza van der Linden \\ Doutor em Engenharia de Produção pela Universidade Federal do Rio Grande do Sul (UFRGS). Professor da \\ Universidade Federal do Rio Grande do Sul (UFRGS) - julio.linden@ufrgs.br
}

\section{Resumo}

A abordagem etnográfica tem sido cada vez mais adotada em diferentes etapas do processo de design. No início do projeto, pode assumir um papel estratégico e auxiliar na identificação das necessidades dos usuários. Contudo, a Antropologia (área de origem da etnografia) e o Design diferem em termos de objetivo, do tempo empregado para a pesquisa e do conhecimento adquirido durante a formação dos profissionais e, diante disso, entende-se que a abordagem etnográfica deve passar por adaptações para ser aplicada na área do Design. Com este estudo, buscou-se entender como a abordagem etnográfica se apresenta quando é aplicada no início do processo de design, para identificar as necessidades dos usuários. Para tanto, investigouse a pesquisa etnográfica como método científico e a pesquisa de campo com usuários no processo de design. Como resultado, foram definidos os principais aspectos da abordagem etnográfica, quando aplicada ao design, e identificados alguns métodos de pesquisa com usuários influenciados por tais aspectos. Percebeu-se que a abordagem etnográfica tem sido utilizada em empresas por meio da aplicação de métodos que apresentam os aspectos identificados neste estudo. Os métodos são fundamentados, principalmente, na observação em contexto e na conversação, a partir dos quais se busca compreender mais profundamente como os usuários se comportam na realidade e, assim, identificar suas necessidades e oportunidades de projetos para atendê-las.

Palavras-chave: Necessidades dos usuários. Pesquisa etnográfica. Insights. Processo de design.

\begin{abstract}
The ethnographic approach has been increasingly adopted at different stages of design process. At the beginning of the project, it can assume a strategic role and assist in identifying the users' needs. However, Anthropology (area of ethnography origin) and Design differ in terms of purpose, time spent for research and knowledge acquired during a training of professionals and, after that, it is understood that the ethnographic approach must passes through adaptations to be applied in the area of Design. With this study, we sought to understand how an ethnographic approach presents itself when it is applied at the beginning of the design process to identify the users' needs. Therefore, they were investigated the occurrence of ethnographic research as a scientific method and the field research with users in process design. As result, were defined the main aspects of ethnographic approach applied to design and identified some users' research methods influenced by such aspects. It was realized that the ethnographic approach has been used in companies through the application of methods that presents the aspects identified in this study. These methods are based, mainly, on observation in context and conversation, in which it is sought to understand deeply how users behave in reality and thus, identifying their needs and projects to meet them.
\end{abstract}

Keywords: Users' needs. Ethnographic research. Insights. Design process.

Recebido em: 09/04/2017

Aceito em: 28/11/2017 


\section{INTRODUÇÃO}

A etnografia pode desempenhar importante papel no processo de design, apoiando na percepção da visão de mundo de grupos de diferentes contextos socioculturais. Tem como principal característica a inserção do pesquisador no contexto de vivência de um grupo de pessoas para observá-las, encontrar padrões e delinear sua cultura, reunindo informações sobre o que as pessoas fazem, o que dizem e como trabalham, por meio de documentos, anotações, fotos, etc. (ROGERS; SHARP; PREECE, 2013). A etnografia faz parte do processo de construção de conhecimento em Ciências Sociais por possibilitar a experiência de interação social e cultural entre o pesquisador e as sociedades estudadas por ele (ROCHA; ECKERT, 2008).

Os designers têm buscado na abordagem etnográfica suporte para se aproximar dos usuários de forma mais adequada, a fim de compreender seu comportamento e identificar suas reais necessidades no início do projeto. Atualmente, é comum encontrar psicólogos e antropólogos trabalhando em equipe com designers, segundo Suri (2011, p. 17), "compartilhando e adaptando métodos, integrando insights, gerando, evoluindo e implementando ideias". Observar e entrevistar usuários nos ambientes em que vivem tem sido uma atividade amplamente estabelecida no Design, conhecida no mundo dos negócios por "estilo de pesquisa etnográfico" - forma valiosa para se obter insights do consumidor. Esse tipo de pesquisa tende a gerar insights de comportamentos, crenças e metas de um grupo de pessoas, os quais podem ser explorados conforme os produtos que uma empresa está pensando em lançar, conduzindo a inovação a partir do ponto de vista do usuário (KUMAR; WHITNEY, 2007).

No processo de design, a etnografia constitui a base de vários métodos centrados no ser humano, fundamentados em observação, conversação e reflexão. Tais métodos possibilitam que o designer entenda o que as pessoas fazem e porque fazem, considerando o seu ponto de vista e os elementos que podem influenciar nas suas ações e comportamentos. Comumente a etnografia é adotada nas fases iniciais do processo de design, apresentando-se em uma linha que tende mais ao aspecto exploratório, em que a equipe vai investigar o comportamento de um grupo de pessoas. Contudo, também pode assumir caráter avaliativo nas fases finais do processo, sendo utilizada para testar um produto ou protótipo (SANDERS, 2004; MARTIN; HANINGTON, 2012). Pelo fato de acontecer em ambientes reais, a etnografia torna a avaliação do produto mais próxima da realidade (SANDERS, 2004). 
Embora a etnografia tenha sido amplamente praticada no processo de design, cabe reconhecer que a Antropologia (área de origem da etnografia) e o Design possuem objetivos distintos: na Antropologia, o interesse do etnógrafo é compreender o comportamento humano exatamente como se reflete nas formas de vida de diversas comunidades; já, no Design, o designer está interessado em desenvolver artefatos que sejam utilizados para dar suporte às atividades dessa comunidade (BLOMBERG et al., 1993). Segundo Bichard e Gheerawo (2010), o Design está mais interessado em insights criativos do que na compreensão expansiva de cada aspecto da vida do usuário.

As áreas também divergem em outros aspectos. A Antropologia é, tradicionalmente, caracterizada pelo longo tempo de que o pesquisador dispõe para imergir na cultura do usuário. Diferentemente do Design, no qual o ciclo de desenvolvimento de um produto ou serviço é de rápida duração, e o prazo que o designer tem, para realizar a pesquisa com o usuário, é limitado em meio a outras pesquisas realizadas ao longo do processo (BLOMBERG et al., 1993; BOZTEPE, 2007). Outro aspecto que deve ser observado é que o profissional de Design possui formação distinta do antropólogo. Para os etnógrafos com formação em Ciências Sociais, a prática etnográfica também requer conhecimento histórico e teórico específico da área, pois qualifica o etnógrafo a questionar, interpretar e situar o que ele vê dentro de um amplo contexto cultural (GOODMAN; KUNIAVSKY; MOED, 2012; SULLIVAN, 2004 apud SANDERS, 2004).

Hanington (2003) afirma que, ainda que os designers não possuam o conhecimento dos profissionais de outras áreas que realizam a pesquisa, sua participação na pesquisa com usuários atende pelo menos a dois propósitos-chave: i) o designer possui conhecimento para interpretar a informação no contexto onde está acontecendo (seja uma preferência expressada por um usuário individual ou um padrão de comportamento) e balanceá-la com as possibilidades criativas e as limitações do projeto. Isto é, o designer filtra as informações conforme os fatores envolvidos no projeto, sabendo diferenciar quando deve acatá-las ou quando deve considerar as entrelinhas do que foi percebido. ii) A imersão no processo de pesquisa e o contato direto com usuários produzem um senso de empatia entre o designer e o usuário, o que leva o designer a se sentir responsável por corresponder às expectativas das pessoas que contribuíram para a pesquisa.

Comumente, o designer vai a campo e realiza a pesquisa com usuários; em outros casos, uma equipe multidisciplinar é responsável pelo projeto, e cada profissional contribui com o seu 
conhecimento e sua visão durante o processo (MASTEN; PLOWMAN, 2003). Nesse caso, os profissionais de outras áreas, como Ciências Sociais, Marketing e Antropologia, são responsáveis pela pesquisa com usuários, eles entendem o comportamento dos usuários e traduzem esse conhecimento em princípios e prescrições de forma que os designers entendam e utilizem (SANDERS, 1999). Neste estudo, o foco está no profissional de Design como responsável pela pesquisa com usuários, que também é denominado de pesquisador, quando as circunstâncias dizem respeito ao processo de design.

Diante do exposto, este artigo buscou identificar como a abordagem etnográfica se apresenta, quando é aplicada no início do processo de design para identificar as necessidades dos usuários. Na fundamentação teórica, investigou-se a pesquisa etnográfica como método científico: como ocorre a pesquisa de campo, quais os principais métodos e materiais utilizados na coleta de dados. Também se investigou como o designer realiza a pesquisa com usuários. Como resultado, foram definidos os principais aspectos da abordagem etnográfica, quando aplicada ao design, e identificados alguns métodos de pesquisa com usuários influenciados por tais aspectos. Percebeuse que a abordagem etnográfica tem sido utilizada nas empresas, por meio da aplicação de métodos que apresentam os aspectos identificados neste estudo. Os métodos são fundamentados, principalmente, na observação em contexto e na conversação, a partir dos quais se busca compreender mais profundamente como os usuários se comportam na realidade, para identificar suas necessidades e oportunidades de projetos.

\section{PROCEDIMENTOS METODOLÓGICOS}

Este artigo buscou identificar como a abordagem etnográfica se apresenta, quando aplicada no início do processo de design, para auxiliar na identificação das necessidades dos usuários. Inicialmente, buscou-se entender a etnografia como método de pesquisa científico, por meio de publicações de áreas diversas, a fim de explorar a pesquisa de campo etnográfica: as etapas, as técnicas, os métodos e os materiais utilizados durante a sua realização. Também se investigou a pesquisa com usuários no processo de design e como a abordagem etnográfica está inserida nesse processo. Essa etapa foi fundamentada em publicações científicas da área do design e em informações coletadas nos sites de empresas que se denominam praticantes da etnografia no desenvolvimento de projetos. 
A partir da revisão de literatura, foram definidos os principais aspectos que a abordagem etnográfica apresenta, quando aplicada no processo de design, a saber: i) possibilita que coleta de dados seja realizada em ambientes reais; ii) possibilita a compreensão dos aspectos de forma global; iii) busca perceber as situações sob o ponto de vista dos usuários; iv) tem um objetivo mais provavelmente exploratório que avaliativo; v) aplica vários métodos simultaneamente; vi) apresenta flexibilidade. Além disso, foi necessário investigar como métodos de pesquisa com usuários possibilitam a prática da abordagem etnográfica. Para tanto, foram buscadas fontes de referência, cujos processos de design são constituídos por etapas e métodos que atenderam a pelo menos um dos seguintes critérios:

I) Apresentar processo de design com abordagem centrada no humano, pois o processo parte da identificação das necessidades do usuário e evolui em torno disso, entrando em concordância com o objetivo deste estudo.

II) Apresentar processo de design voltado à inovação de produtos ou serviços, que utiliza a etnografia na condução da pesquisa com usuários. Observou-se, na revisão de literatura, que grande parte das referências que propõem a utilização da etnografia, durante a pesquisa com usuários, apresenta a inovação como tema central ou secundário.

Figura 1 - Fontes de referência utilizadas no estudo.

\begin{tabular}{|c|c|c|c|c|}
\hline \multicolumn{2}{|c|}{ TIPO DE FONTE } & \multirow{2}{*}{$\begin{array}{l}\text { NOME DA FONTE } \\
101 \text { Design Methods }\end{array}$} & \multirow{2}{*}{$\begin{array}{l}\text { AUTORIA } \\
\text { Vijay Kumar }\end{array}$} & \multirow{2}{*}{$\begin{array}{l}\text { ANO } \\
2013\end{array}$} \\
\hline [1] & Livro & & & \\
\hline [3] & Livro & Universal Methods of Design & Martin e Hanington & 2012 \\
\hline [4] & Livro & Delft Design Guide & TU Delft Industrial Design & 2013 \\
\hline [6] & Manual & Innovating For People & Luma Institute & 2012 \\
\hline [7] & Manual & Human-Centered Design & IDEO & 2010 \\
\hline [8] & Manual & Collective Action Tookit (CAT) & Frog & 2012 \\
\hline
\end{tabular}

Fonte: elaborado pelos autores. 
A figura 1 apresenta as seis fontes de referência selecionadas, das quais foram retirados os métodos de pesquisa com usuários ${ }^{1}$ que possuem os principais aspectos da abordagem etnográfica aplicada no processo de design, definidos neste estudo.

Os métodos selecionados foram agrupados conforme características semelhantes referentes a sua proposta principal: i) métodos para observação em contexto; ii) métodos de auto documentação; iii) métodos para desenvolvimento de atividades e; vi) métodos de investigação de artefatos. Os métodos de cada grupo são descritos a fim de esclarecer como a abordagem etnográfica ocorre no processo de design.

\section{A ABORDAGEM ETNOGRÁFICA COMO MÉTODO CIENTÍFICO}

A etnografia é vista em muitas áreas como um estilo de pesquisa que abrange métodos qualitativos distintos, combinados conforme necessário, para trazer à tona respostas e ideias (ANGROSINO, 2009). Para Peirano (1992, p. 3), “[...] a etnografia não é apenas um método, mas uma forma de ver e ouvir, uma maneira de interpretar, uma perspectiva analítica, a própria teoria em ação". A pesquisa qualitativa é composta por um conjunto de práticas interpretativas que transformam o mundo real em uma série de representações através de notas de campo, entrevistas, conversas, fotografias, gravações e notas particulares do pesquisador sobre sua experiência; os pesquisadores estudam as coisas em seus ambientes naturais com o intuito de interpretar os fenômenos e os significados que as pessoas atribuem aos mesmos (DENZIN; LINCOLN, 2011).

Vários autores afirmam que a etnografia, como método de pesquisa, fundamenta-se na observação participante - que é caracterizada pela prática da observação juntamente à conversação ou entrevista não estruturada. A observação e a conversação são consideradas métodos complementares, visto que a observação fornece dados visuais ao pesquisador e a conversação possibilita respostas diretas dos usuários, esclarecendo dúvidas latentes dos pesquisadores ou confirmando dados observados (ANGROSINO; ROSENBERG, 2011; KENSING; BLOMBERG, 1998; JORDAN, 1996; STANTON; BABER, 1996; ROCHA; ECKERT, 2008; ROGERS; SHARP; PREECE, 2013). A observação participante é uma habilidade complexa que requer formação,

\footnotetext{
${ }^{1} \mathrm{O}$ estudo completo envolveu 337 métodos de pesquisa com usuários (FIALHO, 2014), dentre os quais, 14 apresentam abordagem etnográfica e estão descritos neste artigo.
} 
experiência e reflexão contínua sobre o processo, devendo ser praticada, levando em consideração as responsabilidades éticas do pesquisador (DENZIN; LINCOLN, 2011; JORDAN, 1996).

A observação participante possibilita que o pesquisador perceba a diferença entre o que as pessoas dizem e o que fazem - uma das principais motivações para estudos que envolvem a observação do comportamento humano na área de Antropologia (BLOMBERG et al., 1993). No entanto, apesar da observação participante ser a principal prática em métodos etnográficos, existe diversas formas de observação, o que possibilita que o pesquisador escolha a mais adequada a sua pesquisa, bem como oferece possibilidades para mudança de estratégia de observação no decorrer da pesquisa (BLOMBERG et al., 1993). Essas eventuais mudanças de estratégias evidenciam a natureza flexível que a pesquisa etnográfica deve possuir. À medida que o pesquisador adquire experiência em determinada situação, desenvolve a capacidade de moldar o andamento da pesquisa sem prejudicar a execução da mesma (ANGROSINO, 2009; ROGERS; SHARP; PREECE, 2013).

A observação em si é "um ato de perceber um fenômeno, muitas vezes com instrumentos, e registrá-lo com propósitos científicos" (ANGROSINO, 2009, p. 74), em que o pesquisador engaja-se em uma experiência para perceber contrastes sociais, culturais, e históricos de um grupo (ROCHA; ECKERT, 2008). Independentemente do tipo de observação escolhida para a pesquisa, alguns aspectos principais costumam ser considerados pelos pesquisadores: as pessoas, como acontecem as relações entre elas, o contexto onde estão, os eventos, as atividades que realizam e os objetos (BLOMBERG et al., 1993; ROGERS; SHARP; PREECE, 2013). Esse tipo de pesquisa enfoca a interação dos indivíduos com todos os elementos do contexto (GERHARDT; SILVEIRA, 2009).

Durante a pesquisa de campo, os registros podem ser feitos por meio de gravação de imagens e áudios, com a utilização de uma extensa gama de produtos tecnológicos disponíveis atualmente, como câmeras, smartphones, tablets, gravadores de áudio, etc. (ANGROSINO; ROSENBERG, 2011). A gravação de vídeo tem um papel importante na coleta de dados, pois "[...] o vídeo preserva os aspectos visuais dos dados que muitas vezes são perdidos durante a transição de conversações" (GIBBS, 2009, p. 17). A gravação possibilita que o pesquisador analise o material coletado quantas vezes julgar necessário, como também discuta sobre os resultados com os outros membros da equipe. Dessa forma, o vídeo possibilita que outros membros interpretem a situação 
com seu repertório peculiar, acessando os dados livres de alteração ou interpretação prévia (ANGROSINO, 2009; BLOMBERG et al., 1993; ROGERS; SHARP; PREECE, 2013).

Por outro lado, as gravações de imagens ou áudios podem gerar problemas se forem produzidas em grandes quantidades. Os pesquisadores terão muitos dados para analisar e nem sempre o período de tempo é satisfatório para gerar boas análises; além disso, material em excesso pode provocar dificuldade para o pesquisador localizar um trecho de seu interesse. As anotações podem facilitar a organização dos vídeos, auxiliando na localização de momentos específicos da pesquisa, como também pode complementar as informações com observações pontuais (BLOMBERG et al., 1993; ROGERS; SHARP; PREECE, 2013).

O outro método utilizado na observação participante é a conversação que é um tipo de entrevista não estruturada de caráter exploratório e investigativo, é de natureza aberta, similar a uma conversa, e diz respeito à interação com pessoas relativamente desconhecidas (ANGROSINO, 2009; BLOMBERG; BURELL; GUEST, 2002; ROGERS; SHARP; PREECE, 2013). A conversação possibilita que o pesquisador elucide, com indagações informais, questionamentos que podem surgir durante a observação, mesmo porque não poderia confiar na sua intuição para discernir as atitudes dos pesquisados (ANGROSINO; ROSENBERG, 2011). A conversação também pode revelar aspectos do usuário desconsiderados pelo pesquisador anteriormente (LI, 2008; ROGERS; SHARP; PREECE, 2013).

Durante muito tempo, a pesquisa qualitativa foi alvo de questionamentos referentes a sua relevância e validade. Devido ao caráter qualitativo, a pesquisa etnográfica é considerada subjetiva pelos vários fatores que podem influenciar a imparcialidade do pesquisador durante a pesquisa e, consequentemente, a integridade dos dados (O'GRADY, J.; O'GRADY, K., 2006). Por essa razão, vários autores sugerem a utilização de uma combinação de métodos na coleta de dados, para que o pesquisador confronte os resultados obtidos a partir das diferentes perspectivas que cada método pode oferecer. Tal procedimento é denominado de triangulação e tem a finalidade de abranger a maior amplitude possível ao descrever, explicar e compreender o objeto de estudo, sendo, pois, sugerida por diversos autores para análise do material coletado (ANGROSINO, 2009; GOLDENBERG, 2004; O'GRADY, J., O'GRADY, K., 2006; ROGERS; SHARP; PREECE, 2013; SANDERS, 1992). 


\section{O PESQUISADOR NO CONTEXTO DO USUÁRIO}

A abordagem etnográfica tem sido utilizada no Design por meio dos métodos e técnicas que constituem processos de design centrados no humano. Kumar e Whitney (2003) apresentam dois tipos de pesquisa com usuários fundamentados em práticas etnográficas. A primeira é a pesquisa focada na cultura que identifica padrões e leva a descobertas sobre a cultura de um povo, cuja base provém da imersão: método etnográfico em que o pesquisador permanece no contexto do grupo estudado por um período mais extenso em relação a métodos de aplicação mais rápida. O pesquisador observa, conversa e realiza diversas atividades para extrair informações dos usuários de diferentes formas e acompanha o grupo em atividades que lhes são cotidianas (figura 2 e figura 3). "[...] O pesquisador deve estar apto a descrever não apenas o que os membros de uma cultura fazem, mas a experiência deles ao fazer algo, e como essas ações se encaixam nas suas rotinas" (DOURISH, 2001, p. 59).

Figura 2 - Mulheres realizando suas atividades cotidianas em comunidade.

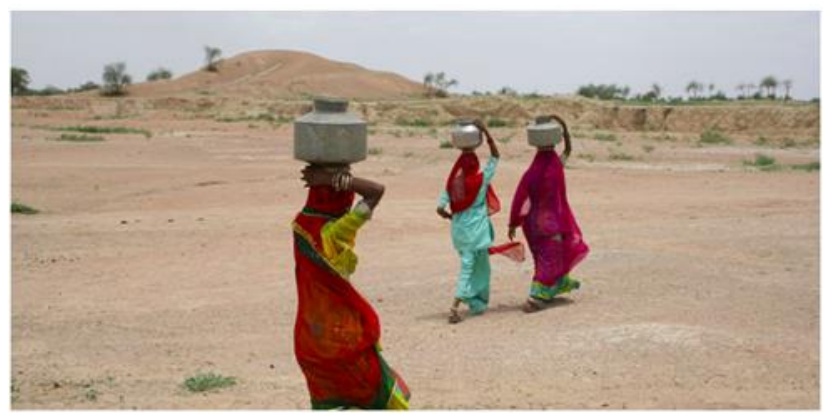

Fonte: www.ideo.com

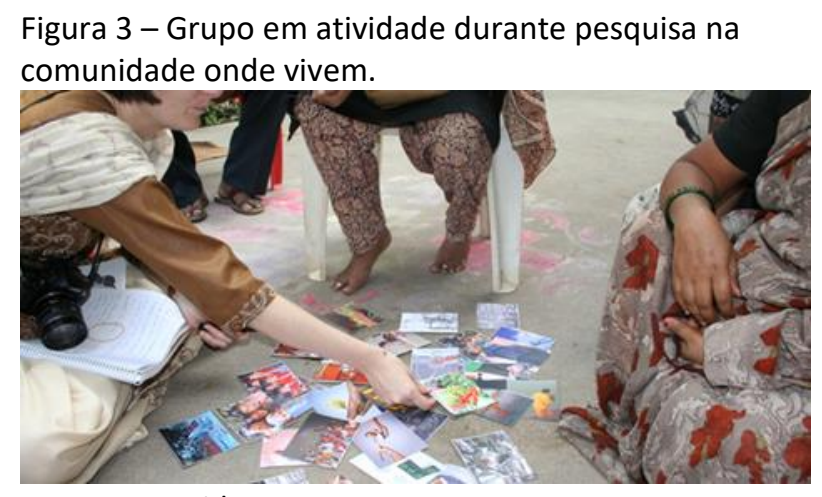

Fonte: www.ideo.com

Em outro contexto, mas utilizando a mesma estratégia, estão as corporações multinacionais e as Organizações Não Governamentais (ONGs) que trabalham com o Design para a Base da Pirâmide (BOP) (WHITNEY; KELKAR, 2004). As corporações trabalham juntamente com ONGs, com o governo local e com comunidades de baixa renda financeira (PRAHALAD; HART, 2002). Tais comunidades são constituídas pelas pessoas "mais pobres do mundo", geralmente moradores da África, Europa Oriental, América Latina e Ásia (CONFEDERATION OF DANISH INDUSTRIES, 2007).

Empresas que exploram as oportunidades do BOP, como Nike, Johnson \& Johnson, Procter \& Gamble, entre outras (SIMANIS; MILSTEIN, 2012), oferecem soluções às comunidades na forma 
de novos produtos ou negócios que podem fornecer produtos e serviços a preços acessíveis, cujas ideias surgiram da pesquisa de campo realizada para observação das atividades cotidianas das pessoas que pertencem às comunidades beneficiadas.

Sendo um método de pesquisa que demanda maior tempo para ser aplicado, a prática da imersão é restrita a certos tipos de projetos. Com os prazos cada vez mais curtos para o desenvolvimento de um projeto de design, torna-se difícil a aplicação da imersão de forma eficiente (KUMAR; WHITNEY, 2007). A maioria das pesquisas de campo realizadas no processo de design é breve: podem ser transformadas em uma tarde no shopping, uma semana frequentando um restaurante, uma quinzena em algum país com outra cultura, etc. (SALVADOR; BELL; ANDERSON, 1999). Diante do grande número de possíveis projetos, vários métodos têm sido desenvolvidos e adaptados para atender a requisitos de diferentes situações de projeto.

A segunda proposta que Kumar e Whitney (2003, p. 50) apresentam tem como objetivos "identificar oportunidades ainda não atendidas e aumentar a velocidade e o êxito da inovação em novas regiões geográficas" - é a pesquisa com foco na atividade. A proposta também busca conhecer a cultura de um grupo, contudo, focando na atividade dos usuários. Ou seja, tem foco "nas atividades das pessoas, quando elas estão usando um produto ou um serviço que uma empresa quer desenvolver" (KUMAR; WHITNEY, 2003, p.52). O foco não está no shampoo (produto), por exemplo, mas sim na experiência que envolve o uso do produto, como usar o shampoo para se arrumar para uma festa, preparar-se para uma festa (KUMAR; WHITNEY, 2007). A pesquisa também acontece no contexto dos usuários (figura 4), mas requer menor tempo para ser realizada, já que o pesquisador acompanha atividades específicas do usuário.

Durante a pesquisa com foco na atividade, o pesquisador deve atentar para o desempenho da atividade em um nível macro, observando da forma mais natural possível todas as atividades que cercam o uso do produto, e não apenas o produto em si (KUMAR; WHITNEY, 2007). Os autores citam uma situação de pesquisa realizada para uma empresa que desenvolve aparelhos de cozinha, na qual os pesquisadores observaram famílias não apenas durante as refeições, mas durante o planejamento, a compra de mantimentos, durante as ações de preparar, servir e comer as refeições e durante a realização da limpeza (KUMAR; WHITNEY, 2003). 
Figura 4 - Pesquisador observa pessoa em atividade.

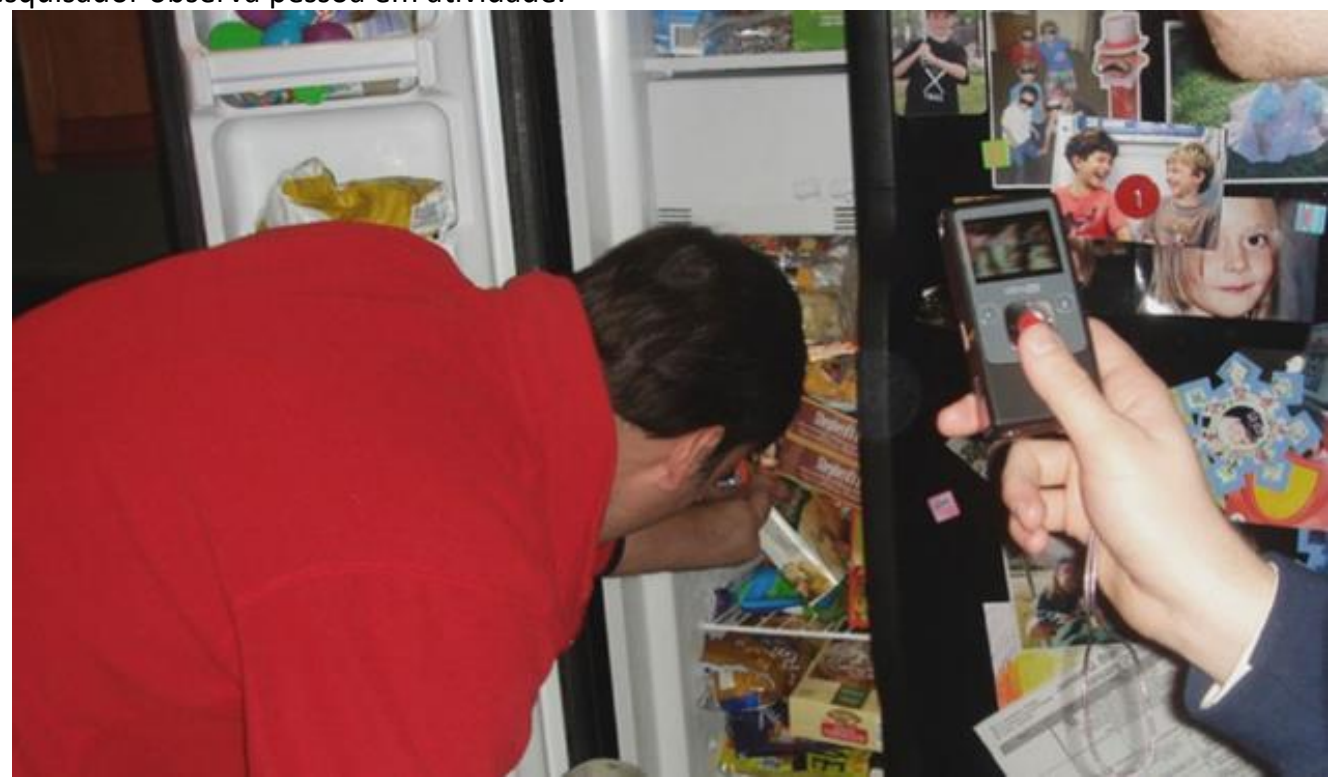

Fonte: www.beyonddesignchicago.com

A pesquisa apoia-se na utilização de recursos tecnológicos para pesquisa de campo, como pequenas câmeras de vídeo e afins para a condução de vídeo etnografia, câmeras descartáveis para a criação de um diário de fotos (auto documentação), e para o tratamento dos dados, como softwares que auxiliam no armazenamento e organização dos dados e na análise, extraindo insights para o desenvolvimento de produtos e serviços (KUMAR; WHITNEY, 2003). A pesquisa com foco na atividade requer a participação de um menor grupo de usuários, e é apenas um dos exemplos encontrados na literatura, cuja finalidade é balancear o tempo disponível para a realização da pesquisa com usuários numa situação de projeto.

Assim como a pesquisa focada na atividade, outras abordagens, ferramentas, métodos e técnicas têm sido desenvolvidos para adequar os preceitos etnográficos à pesquisa com usuários no processo de design (HANINGTON, 2003). São estruturas que guiam a pesquisa em campo com a finalidade de identificar necessidades e oportunidades de um grupo de pessoas. "[...] Aqueles que defendem a adoção da etnografia no design para os mercados globais, também apontam para a necessidade de ferramentas eficientes e estruturas para a realização, análise e apresentação de pesquisa do usuário" (BOZTEPE, 2007, p. 517).

Uma ferramenta bastante conhecida é o manual Human-Centered Design - HCD (IDEO, 2010), produzido pela empresa de Design IDEO, que desenvolve projetos utilizando uma 
abordagem centrada no ser humano, com a finalidade de auxiliar a inovação e o crescimento em organizações públicas e privadas. O objetivo da ferramenta é auxiliar as pessoas e os voluntários a compreenderem as necessidades de uma comunidade de uma nova maneira, considerando a sustentabilidade financeira na busca de soluções inovadoras para atender às necessidades das pessoas da comunidade (IDEO, 2010). O manual pode ser utilizado por pessoas que não são especializadas em pesquisa ou em design.

O manual Collective Action Toolkit - CAT (FROG DESIGN, 2012), desenvolvido pela Frog Design, segue o mesmo princípio. É uma empresa de design que, assim como a Ideo, se baseia no design Centrado no Humano para realizar seus projetos, buscando guiar os pesquisadores na solução de problemas da comunidade estudada. Além dos manuais e materiais afins, disponibilizados por empresas de design, o designer também pode utilizar métodos avulsos, compilados em livros e sites que abordam a pesquisa com usuários e que possibilitam a adaptação dos métodos ao contexto do projeto.

\section{A ABORDAGEM ETNOGRÁFICA APLICADA AO DESIGN}

Considerando as diferenças existentes entre as áreas de Antropologia e de Design, foram definidos os principais aspectos que a abordagem etnográfica apresenta, quando aplicada no início do processo de design, com o objetivo de identificar as necessidades dos usuários (figura 5). Percebeu-se que esse tipo de abordagem: i) possibilita que a coleta de dados seja realizada em ambientes reais; ii) possibilita a compreensão dos aspectos de forma global; iii) possibilita a percepção das situações sob o ponto de vista dos usuários; iv) tem um objetivo, provavelmente, mais exploratório que avaliativo; v) possibilita a aplicação de vários métodos simultaneamente e; vi) apresenta flexibilidade. Explicadas a seguir:

I) Possibilita que coleta de dados seja realizada em ambientes reais: acredita-se que o contexto de vivência dos usuários permite que os mesmos se sintam "mais confortáveis e comunicativos" (KUMAR, 2012, p. 111), e que façam referências a seus objetos pessoais enquanto conversam com o pesquisador ou, ainda, que eles próprios coletem os dados, auxiliando o andamento da pesquisa. Assim, a coleta de dados pode ser conduzida pelo pesquisador no ambiente real dos usuários ou pelos próprios usuários dentro do cenário no qual estão inseridos. Os usuários coletam os dados por meio da aplicação de métodos de auto documentação, 
possibilitando igualmente que o pesquisador tenha acesso ao contexto habitual dos mesmos (BLOMBERG; BURELL; GUEST, 2002). Os métodos de auto documentação são indicados quando o pesquisador, por alguma razão, não pode realizar a pesquisa.

II) Possibilita a compreensão dos aspectos de forma global: a pesquisa deve possibilitar uma visão sistêmica do contexto pesquisado, uma vez que todos os aspectos pertencentes ao ambiente ou atividades realizadas podem influenciar o comportamento do usuário. As atividades não devem ser estudadas de forma isolada, visto que ocorrem em paralelo a outras atividades, conectadas a um contexto de espaço e tempo. Dessa forma, os aspectos contidos no contexto do usuário devem ser considerados em conjunto, a fim de evitar uma percepção limitada e ilusória da atividade (BLOMBERG; BURELL; GUEST, 2002).

III) Busca perceber as situações sob o ponto de vista dos usuários: a pesquisa etnográfica busca descobrir o ponto de vista das pessoas de um grupo que está sendo estudado e que, geralmente, são potenciais consumidores ou usuários finais de produtos novos ou já existentes. O pesquisador deve tentar compreender os acontecimentos e ações a partir da perspectiva das pessoas, ou seja, como elas falam sobre o que está acontecendo; o que é importante e significativo para elas e o que não é; que recursos no seu ambiente elas usam; como elas resolvem seus problemas e como realizam seu trabalho; descobrir o que não foi identificado em estudos prévios e o que os participantes não falam (JORDAN, 1996).

IV) Tem um objetivo, provavelmente, mais exploratório que avaliativo: no contexto do Design, uma pesquisa avaliativa é caracterizada pelo objetivo de avaliar conceitos, protótipos, produtos, tecnologias, etc. O foco desse tipo de pesquisa gira em torno do produto que está sendo desenvolvido ou redesenhado. Já, as pesquisas com objetivo exploratório são voltadas à compreensão do comportamento das pessoas, para identificar suas necessidades, possibilitando maior percepção para ideias de projetos. Considerando a atividade de investigação dos aspectos culturais de um grupo, compreende-se que a abordagem etnográfica tem um objetivo, provavelmente, mais exploratório que avaliativo (SANDERS, 2002).

V) Aplica vários métodos simultaneamente: a pesquisa com abordagem etnográfica combina uma série de métodos para coletar os dados, incluindo a observação e as formas abertas de entrevistas e questionamentos. O objetivo de mesclar mais de um método na coleta de dados é 
minimizar as eventuais influências peculiares a cada método, assim, os dados coletados com a aplicação de um método podem confirmar os coletados com a aplicação de outro (SANDERS, 1992).

VI) Apresenta flexibilidade: a abordagem etnográfica é aberta a mudanças e aperfeiçoamento ao longo do processo, aperfeiçoa a prática para observações futuras (SANDERS, 2004). Logo, alguns acontecimentos durante a pesquisa podem alterar o planejamento inicial para que os objetivos sejam alcançados.

Figura 5 - Caracterização da abordagem etnográfica aplicada ao design.

\section{A ABORDAGEM ETNOGRAFICAAPLICADAAO DESIGN}

\begin{tabular}{|c|c|c|c|c|c|}
\hline $\begin{array}{l}\text { Possibilita a } \\
\text { coleta dos dados } \\
\text { em ambientes } \\
\text { reais }\end{array}$ & $\begin{array}{l}\text { Possibilita a } \\
\text { compreensão } \\
\text { dos aspectos de } \\
\text { forma global }\end{array}$ & $\begin{array}{l}\text { Possibilita a } \\
\text { percepção das } \\
\text { situações sob o } \\
\text { ponto de vista } \\
\text { dos usuánios }\end{array}$ & $\begin{array}{l}\text { Tem objetivo, } \\
\text { provavelmente, } \\
\text { mais exploratório } \\
\text { que avaliativo }\end{array}$ & $\begin{array}{l}\text { Possibilita a } \\
\text { aplicação de } \\
\text { vários métodos } \\
\text { ao mesmo tempo }\end{array}$ & $\begin{array}{l}\text { Apresenta } \\
\text { flexibilidade }\end{array}$ \\
\hline
\end{tabular}

Fonte: elaborado pelos autores.

\section{A abordagem etnográfica em métodos de pesquisa com usuários}

A literatura apresenta inúmeros métodos de pesquisa com usuários, dentre os quais, alguns são vistos como métodos com influência da etnografia, cujos aspectos coincidem com os aspectos da abordagem etnográfica aplicada ao design. Percebeu-se que, segundo a descrição, a prática desse tipo de método possibilita que o designer realize pesquisa exploratória em ambientes reais para compreender o comportamento dos usuários e identificar suas necessidades. $\mathrm{O}$ fato de ir ao contexto dos usuários possibilita que o designer perceba as situações sob a ótica do usuário e tenha uma visão sistêmica, ou seja, do usuário, de suas ações, dos elementos envolvidos no ambiente e como todos se relacionam. Além disso, são métodos fundamentados, ao menos, pela observação e conversação, constituindo duas fontes de coleta para confrontar as informações obtidas. Também são flexíveis - podem ser adaptados conforme o andamento da pesquisa, caso seja necessário.

Para este estudo, foram encontrados alguns métodos com tais características, explicados adiante. Esses métodos apresentam semelhanças e, por isso, foram agrupados conforme sua proposta principal (figura 6): i) métodos para observação em contexto; ii) métodos de auto 
documentação; iii) métodos para desenvolvimento de atividades e; vi) métodos de investigação de artefatos.

Figura 6 - Métodos de pesquisa com usuários com abordagem etnográfica.

\begin{tabular}{|l|l|l|l|}
\hline \multicolumn{3}{c|}{ MÉTODOS DE PESQUISA COM USUÁRIOS COM ABORDAGEM ETNOGRAFICA } \\
\hline Observação em contexto & Auto documentação & Desenvol. de atividades & Investigaçäo de artefatos \\
\hline Artifact Analysis & User Observation & Diary Studies & Field Activity \\
\hline Touchstone Tours & Field Visit & Joumaling & Image Sorting \\
\hline & Fly-on-the-wall Observation & Experience Sampling Method & Buy a Feature \\
\hline & Shadowing & & \\
\hline & Ethnographic Interview & \\
\hline
\end{tabular}

Fonte: elaborado pelos autores.

I) Na observação em contexto, o pesquisador frequenta o contexto dos usuários e os observa realizando atividades cotidianas, sem interferência. $O$ pesquisador pode solicitar a realização de uma atividade geral sem nenhum direcionamento ou relacionada a um tema específico. O objetivo é observar o comportamento das pessoas, buscando entender a relação entre as atitudes e o contexto e, ao mesmo tempo, criando empatia pelos usuários.

O método User Observation (KUMAR, 2012; MARTIN; HANINGTON, 2012) é a observação em si, quando envolve conversação é caracterizada como observação participante. O método Field Visit (KUMAR, 2012) é similar, o pesquisador vai ao contexto dos usuários, observa-os realizando suas atividades e, ao mesmo tempo, questiona-os sobre o que está sendo observado; é uma forma de aproximação entre pesquisador e usuário, que também busca a construção da empatia. No método Fly-on-the-wall Observation (LUMA INSTITUTE, 2012; MARTIN; HANINGTON, 2012), o pesquisador não participa nem interfere, apenas observa e escuta o usuário desenvolvendo atividades. Pode ser realizado com o usuário consciente de que está sendo observado (o que pode alterar seu comportamento), ou sem o seu conhecimento (com as devidas permissões).

No método Shadowing (MARTIN; HANINGTON, 2012), o pesquisador acompanha o usuário durante suas atividades, experimentando as situações pelas quais ele passa durante o dia. Nesse tempo, o pesquisador percebe padrões de decisão, coleta ideias em 'primeira mão' e em tempo 
real; pode questionar ou interagir com o usuário durante a atividade, dependendo da conveniência do momento. Quando se trata de um acompanhamento individual ou mais próximo, o usuário sabe da pesquisa. No entanto, a pesquisa também pode ser realizada em ambientes públicos, envolvendo muitos usuários, não sendo necessariamente obrigatório que os usuários saibam da pesquisa.

No método Ethnographic Interview (KUMAR, 2012), o pesquisador conversa com o usuário sobre as atividades que ele realiza, buscando entender as experiências sob a perspectiva deste. No método Contextual Inquiry (LUMA INSTITUTE, 2012; MARTIN; HANINGTON, 2012), o pesquisador observa e entrevista o usuário no seu ambiente de trabalho e pode observar a estrutura de trabalho como realmente é, percebendo o que está além das experiências pessoais do usuário.

II) Na auto documentação, os participantes registram momentos da sua vida sob condições especificadas, sinalizados por alarmes ou não. O objetivo é coletar informações sob a ótica do usuário acerca dos seus sentimentos, pensamentos e comportamentos em momentos específicos. É uma forma de coletar dados indicada em situações nas quais o pesquisador não pode ir ao contexto da pesquisa.

No método Diary Studies (MARTIN; HANINGTON, 2012) e no método Journaling (LUMA INSTITUTE, 2012), o usuário coleta informações em momentos específicos através de registros diários, utilizando um conjunto de ferramentas físicas ou digitais, fáceis de usar e que possua instruções; podem conter imagens, símbolos, desenhos, fotos, textos, adesivos, etc. Em Experience Sampling Method (MARTIN; HANINGTON, 2012), o usuário grava ou documenta algo em momentos específicos, utilizando smartphones com aplicativos específicos que podem ser programados para alarmar na hora de fazer um registro. Comumente, nesse tipo de método, o usuário e o designer conversam posteriormente sobre os dados que foram coletados.

III) Em métodos para desenvolvimento de atividades, o pesquisador propõe atividades específicas que envolvem as pessoas para observar suas reações e decisões durante a realização dessas atividades, auxiliando-as, também, na comunicação de ideias abstratas.

No método Field Activity (KUMAR, 2012), o pesquisador leva os usuários a algum campo para realizar uma atividade e observar como eles reagem durante esse momento; em seguida, realiza entrevistas para confirmar e esclarecer o que foi observado. No método Image Sorting (KUMAR, 2012), o pesquisador seleciona imagens, agrupando-as de acordo com os critérios 
pertinentes ao tema; as pessoas escolhem, discutem e criam histórias com essas imagens, revelando suas associações e percepções sobre um determinado assunto. O método Buy a Feature (LUMA INSTITUTE, 2012) é realizado em forma de jogo, no qual os participantes expressam decisões de compra, usando dinheiro fictício, com o intuito de comprovar o que realmente valorizam, e não o que dizem que valorizam. Os pesquisadores elaboram "cards" relacionados ao que querem investigar (um produto ou serviço), atribuem valor aos mesmos e dão uma quantia limitada de dinheiro aos participantes que, por sua vez, com o dinheiro limitado, tendem a escolher os itens aos quais dão mais valor. A atividade é realizada individualmente e discutida em conjunto.

IV) Em métodos relacionados à investigação de artefatos, o pesquisador faz uma análise sistemática dos artefatos dos usuários em seu ambiente de vivência para entender seu contexto físico, social e cultural; também pode ser realizada em visitas guiadas. Esse tipo de método dá acesso à organização dos espaços dos usuários.

O método Artifact Analysis (MARTIN; HANINGTON, 2012), realizado no contexto do usuário, consiste num exame sistemático de artefatos, envolvendo materiais, estética e qualidades interativas dos objetos, a fim de entender o contexto físico, social e cultural dos usuários. Sugere-se realizar também uma entrevista para os usuários falarem sobre os artefatos. No método Touchstone Tours (MARTIN; HANINGTON, 2012), o pesquisador faz visitas ao contexto do usuário, para perceber como ele organiza as informações e sistemas através do uso do espaço e artefatos. Geralmente, o usuário mostra-se entusiasmado em mostrar seu espaço e objetos, e o pesquisador pode ir conduzindo a visita com conversas.

É importante destacar que os métodos de pesquisa com usuários descritos são apenas alguns dos vários tipos existentes. Recomenda-se selecionar os métodos de acordo com os objetivos da pesquisa ou do projeto, com a disponibilidade da equipe ou dos participantes, com o tempo disponível para a pesquisa, ou com quaisquer outros aspectos interligados ao projeto em questão.

\section{CONSIDERAÇÕES FINAIS}

A abordagem etnográfica pode ser aplicada no processo de design para atender a diferentes objetivos. Pode ter um objetivo avaliativo, em que o designer observa os usuários utilizando o produto em desenvolvimento e conduz uma pesquisa com participação mais limitada do usuário. 
Nesse caso o foco está no produto, então, os usuários respondem a questões específicas, em tempo determinado, por exemplo. A pesquisa também pode ter objetivo exploratório, na qual o designer observa os usuários realizando suas atividades cotidianas, e estes participam de forma mais livre: o foco está em compreender o comportamento desses usuários e descobrir quais necessidades ainda não foram atendidas, não segue um roteiro, nem se responde a questões sobre algo específico.

Neste estudo, investigou-se como a abordagem etnográfica é aplicada para entender o comportamento das pessoas - a pesquisa tem caráter exploratório e possibilita que o pesquisador investigue um grupo no seu contexto de vivência. Por contexto de vivência entendem-se os lugares onde os usuários realizam suas atividades cotidianas, constituído pelos elementos envolvidos nessas atividades e pela forma como eles se relacionam. A relação entre os indivíduos e tais elementos dá suporte ao pesquisador, para entender o comportamento desse grupo sob a ótica deles mesmos.

Entre as variadas formas de aplicação da etnografia no Design, a imersão é uma das mais abrangentes, pois favorece múltiplas formas de interação entre o pesquisador e o grupo pesquisado, já que o pesquisador permanece na comunidade dos usuários por um período que varia de dias a meses. Na imersão, o pesquisador tem uma convivência mais intensa com o grupo, podendo observar e interagir nas atividades cotidianas, como também propor a prática de diversos métodos no intervalo de tempo em que está imerso no contexto das pessoas. Por tais razões, a pesquisa pode apresentar resultados além dos esperados. Contudo, a imersão demanda maior investimento de recursos por parte da empresa, como tempo e orçamento, o que acaba restringindo a sua prática.

Outra forma de aplicar os preceitos etnográficos é a pesquisa com foco na atividade que, assim como a pesquisa imersiva, possibilita a aplicação de outros métodos, fazendo surgir alternativas viáveis, para estudar o comportamento dos usuários e identificar necessidades que gerem demandas de projetos para atendê-las. Esse tipo de pesquisa se comporta como uma alternativa à imersão, pois requer menos tempo para ser aplicada, podendo ser adotada por empresas e designers de forma mais prática e com menor investimento de recursos. Tanto a pesquisa com foco na cultura (imersão), como a pesquisa com foco na atividade proporcionam 
diferentes formas de aplicar a abordagem etnográfica e podem ser incorporadas em inúmeras situações de projeto de design, conforme as peculiaridades de cada projeto.

Vários autores discutem sobre as lacunas que podem surgir ao se transferir a atividade etnográfica para o Design exatamente como é praticada na Antropologia (BLOMBERG et al., 1993; BICHARD; GHEERAWO, 2010; BOZTEPE, 2007; GOODMAN; KUNIAVSKY; MOED, 2012; SANDERS, 2004), isso porque se trata de diferentes áreas. As principais lacunas abordadas pelos autores são: i) objetivos distintos: o etnógrafo tem como objetivo conhecer o comportamento das pessoas, enquanto o designer busca suprir as necessidades dessas pessoas, percebidas durante atividades, por meio da produção de artefatos; ii) tempo disponível para a pesquisa: na Antropologia, o etnógrafo tem longo tempo disponível para realizar a pesquisa, em oposição ao Design, cujo ciclo de desenvolvimento de um produto ou serviço é de rápida duração; iii) conhecimento da área: o etnógrafo possui conhecimento específico e é capacitado para conduzir a pesquisa de campo e analisar os resultados, enquanto o designer utiliza recursos para auxiliar na realização da atividade, a exemplo das ferramentas.

As ferramentas são uma opção utilizadas por empresas, para auxiliar o designer a realizar a pesquisa com usuários, guiando-o em vários aspectos, como a ordem das atividades desenvolvidas em campo, a forma de coleta e interpretação dos dados, o armazenamento de informações, etc. As ferramentas são, comumente, constituídas por métodos que formam um processo de design completo, ou seja, abrangem desde a pesquisa à implementação do projeto e são flexíveis o suficiente para serem aplicadas em diferentes contextos (ambientes domésticos, de trabalho, de estudo, de lazer, etc.). Dentre tais métodos, estão aqueles pautados nos preceitos etnográficos com o intuito de auxiliar no processo de identificação de necessidades do grupo pesquisado. Algumas empresas de design, como a IDEO e a Frog Design, desenvolveram ferramentas e disponibilizam o conteúdo na Internet, sendo bastante utilizadas no desenvolvimento de diversos tipos de projetos. Além do uso de material auxiliar para conduzir a pesquisa com usuários, a experiência do pesquisador também auxilia na prática da abordagem etnográfica, considerando que, a cada pesquisa realizada, ele (ou ela) lida com variados tipos de acontecimentos, com pessoas de diferentes personalidades e, possivelmente, com projetos de natureza distintos.

De maneira geral, o surgimento de ideias inovadoras para o desenvolvimento de produtos e serviços tem origem na descoberta de necessidades não percebidas ou não atendidas das pessoas. 
Para identificar tais necessidades, busca-se compreender o comportamento de um grupo de pessoas por meio da investigação acerca das suas atividades cotidianas no ambiente em que são realizadas. Nesse contexto, a etnografia contribui para área de Design por possibilitar uma forma amigável de abordar o usuário, buscando conhecer o universo do mesmo sem ultrapassar, nem invadir o seu espaço particular. A prática da abordagem etnográfica proporciona mais naturalidade na condução da pesquisa, tornando mais fluida a aproximação entre o designer e o usuário, como também facilitando a identificação de necessidades que podem ser atendidas com o desenvolvimento de produtos e serviços mais adequados.

\section{REFERÊNCIAS}

ANGROSINO, Michael. Etnografia e Observação Participante. Porto Alegre: Artmed, 2009. ANGROSINO, Michael; ROSENBERG, Judith. Observations on observation: continuities and challenges. In: DENZIN, Norman Kent; LINCOLN, Yvonna Sessions. S. (Ed.). The SAGE Handbook of qualitative research. Los Angeles: Sage Publications, 2011. p. 151-175. Disponível em: < https://in.sagepub.com/sites/default/files/upm-binaries/51018_ch_5.pdf>. Acesso em: 26 set. 2013.

BICHARD, Jo-Anne; GHEERAWO, Rama. The Designer as ethnographer: practical projects from industry. In: CLARKE, Alison (Ed.). Design Anthropology: object culture in the 21st century. Springer Wien: New York, 2010. p. 45-55.

BLOMBERG, Jeanette; BURELL, Mark; GUEST, Greg. An Ethnographic approach to Design. In: JACKO, Julie A.; SEARS, Andrew. (Ed.). The human-computer interaction handbook. Hillsdale: Lawrence Erlbaum Associates, 2002. p. 964-968.

BLOMBERG, Jeanette et al. Ethnographic field methods and their relation to Design. In: SCHULER, Douglas; NAMIOKA, Aki. (Ed.). Participatory Design: principles and practices. Hillsdale: Lawrence Erlbaum Associates, 1993. p. 123-155.

BOZTEPE, Suzan. Toward a framework of product development for global markets: a user-valuebased approach. Design Studies, London, v. 28, n. 5, p. 513-533, set. 2007.

CONFEDERATION OF DANISH INDUSTRIES. Working with the bottom of the pyramid: success in Low-income markets. 2007. Disponível em:

<http://di.dk/sitecollectionDocuments/shop/workingwiththe.pdf>. Acesso em: 12 jun. 2013.

DENZIN, Norman Kent; LINCOLN, Yvonna Sessions. Introduction: the discipline and practice of qualitative research. In: (Ed.). The SAGE handbook of qualitative research. Thousand Oaks, CA: Sage Publications, 2011, p. 1-20. Disponível em: <https://www.sagepub.com/sites/default/files/upm-binaries/40425_Chapter1.pdf>. Acesso em: 26 set. 2018. 
DOURISH, Paul. Where the action is: the foundations of embodied interaction. Cambridge: MIT, 2001.

FROG Design. Creative Toolkit Action. 2012. Disponível em: <https://www.frogdesign.com/wpcontent/uploads/2016/03/CAT_2.0_English.pdf>. Acesso em: 17 mar. 2014.

GERHARDT, Tatiana Engel; SILVEIRA, Denise Tolfo (Org.). Métodos de Pesquisa. Porto Alegre: Editora da UFRGS, 2009. Disponível em:

<http://www.ufrgs.br/cursopgdr/downloadsSerie/derad005.pdf>. Acesso em: 10 mai. 2013.

GIBBS, Graham. Análise de Dados Qualitativos. Porto Alegre: Artmed, 2009.

GOLDENBERG, Mirian. A arte de pesquisar: como fazer pesquisa qualitativa em Ciências Sociais. 8. ed. Rio de Janeiro: Editora Record, 2004.

GOODMAN, Elizabeth; KUNIAVSKY, Mike; MOED, Andrea. Observing the user experience: a practitioner's guide to user research. USA: Elsevier, 2012.

HANINGTON, Bruce. Methods in the making: a perspective on the state of human research in Design. In: Design Issues, [S. I.], v. 19, n. 4, p. 09 -18, 2003.

IDEO. Human-centered Design toolkit. 2010. Disponível em: <www.ideo.com>. Acesso em: 18 out. 2012.

JORDAN, Brigitte. Ethnographic workplace studies. In: SHAPIRO, Dan; TAUBER, Michael; TRAUNMTILLER, Roland (Ed.). The Design of computer supported cooperative work and groupware systems. Amsterdam: North Holland/Elsevier Science, 1996, v. 12, p. 17-42.

KENSING, Finn; BLOMBERG, Jeanette. Participatory Design: issues and concerns. Journal Computer Supported Cooperative Work, Massachusetts, v. 7, n. 3 e 4, p. 167-185, 1998.

KUMAR, Vijay. 101 Design methods: a structure approach for driving innovation in your organization. New Jersey: John Wiley \& Sons, 2012.

KUMAR, Vijay; WHITNEY, Patrick. Daily life, not markets: customer-centered design. Journal of Business Strategy, [S. I.], v.28, n. 4, p. 46-58, 2007. Disponível em: <https://www.emeraldinsight.com/doi/full/10.1108/02756660710760944>. Acesso em: 25 set. 2018. 50-57, 2003.

. Faster, cheaper, deeper user research. Design Management Journal, Boston, v.14, n. 2, p. LI, Jun. Ethical challenges in participant observation: a reflection on ethnographic fieldwork. The Qualitative Report, [S. I.], v. 13, n. 1, p. $100-115,2008$.

LUMA INSTITUTE. Innovating for people: handbook of human-centered Design methods. 1. ed. Pittsburgh: Luma Institute, 2012.

MARTIN, Bella; HANINGTON, Bruce. Universal Methods of Design. USA: Rockport Publishers, 2012. 
MASTEN, Davis; PLOWMAN, Tim M. P. Digital ethnography: the next wave in understanding the consumer experience. Design Management Journal, [S. I.], v. 14, n. 2, p. 75-81, 2003.

O'GRADY, Jennifer Visocky; O'GRADY, Kenneth Visocky. A Designer's research manual: succeed in Design by knowing your clients and what they really need. Berverly: RockPort, 2006.

PEIRANO, Mariza Gomes e Souza. A favor da etnografia. Brasília: Universidade de Brasília, 1992.

PRAHALAD, Coimbatore Krishnarao; HART, Stuart L. The fortune of the botton of the piramid. Strategy + Business, [S. I.], First Quarter, n. 26, 2002.

ROGERS, Yvonne; SHARP, Helen; PREECE, Jennifer. Design de interação: além da interação homemcomputador. Trad. Isabela Gasparini 3 ed. Porto Alegre: Bookman, 2013.

ROCHA, Ana Luiza Carvalho da; ECKERT, Cornelia. Etnografia: Saberes e Práticas. In: PINTO, Céli Regina; GUAZZELLI, Cézar Augusto Barcellos (Org.). Ciências Humanas: pesquisa e métodos. Porto Alegre: Editora da Universidade, 2008.

SALVADOR, Tony; BELL, Genevieve; ANDERSON, Ken. Design ethnography. Design Management Journal, [S. I.], v. 10, n. 4, p. 33-41, 1999.

SANDERS, B.-N. Elizabeth. Converging perspectives: product development research for the 1990s. Design Management Journal, Boston, v.3, n. 4, p. 49-54, 1992. Disponível em: $<$ http://www.maketools.com/articles-papers/ConvergingPerspectives_Sanders_92.pdf $>$. Acesso em: 25 set. 2018.

. Postdesign and participatory culture. In: USERFUL AND CRITICAL: the position of research in Design, 1999, Tuusula. Proceedings... Tuusula: University of Art and Design Helsinki, 1999. Disponível em: <http://www.maketools.com/articlespapers/PostdesignandParticipatoryCulture_Sanders_99.pdf>. Acesso em: 25 set. 2018.

. Special section: ethnography in NPD research how 'applied ethnography' can improve your NPD research process. Visions Magazine, Chicago, 2002. Disponível em: <http://www.maketools.com/articles-papers/EthnographyinNPDResearch_Sanders_02.pdf >. Acesso em: 25 set. 2018.

Ethnography and the Empowerment of Everyday People. 2004. Disponível em: < http://www.maketools.com/articles-papers/EthnographyandEmpowerment_Sanders_04.pdf>. Acesso em: 25 set. 2018.

SIMANIS, Erik; MILSTEIN, Mark. Back to business fundamentals: making "bottom of the pyramid" relevant to core business. Field Actions Science Reports, [S. I.], v. 6, n. 4, p. 82-88, 2012.

STANTON, Neville; BABER, Chris. Factors affecting the selection os methods and techniques prior to conducting a usability evaluation. In: JORDAN, Patrick W.; THOMAS, Bruce; WEERDMEESTER, Bernard A.; MCCLELLAND, Ian Lyall. (Ed.). Usability evaluation in industry. Great Britain: T. J. Press, 1996. p. 39-48. 
SURI, Jane Fulton. Poetic observation: what designers make of what they see. In: Design Anthropology: object culture in the 21st century. Springer Wien: New York, 2011, p. 18-32.

WHITNEY, Patrick; KELKAR, Anjali. Designing for the base of the pyramid. Design Management Review, Boston, v.15, n. 4, p. 41-47, 2004. 